\title{
A Flexible Approach to the Multidimensional Model: The Fuzzy Datacube
}

\author{
Miguel Delgado ${ }^{a}$, Carlos Molina $^{\mathrm{a}}$, Daniel Sánchez ${ }^{\mathrm{a}}$, Lázaro Rodriguez Ariza ${ }^{\mathrm{b}}$, M. Amparo Vila ${ }^{\mathrm{a}}$ \\ ${ }^{a}$ Universidad de Granada, Dpto. de Ciencias de la Computación e I.A., E.T.S.I. de Informática \\ C/ Periodista Daniel Saucedo Aranda s/n \\ 18071 Granada, Spain \\ \{mdelgado, carlosmo, daniel, vila\}@decsai.ugr.es \\ ${ }^{\mathrm{b}}$ Universidad de Granada, Depto. Economía Financiera y Contabilidad, \\ Faculty of Economics and Business Sciences \\ Campus Universitario de la Cartuja, 18071 Granada, Spain \\ lazaro@ugr.es
}

\begin{abstract}
As a result of the use of OLAP technology in new fields of knowledge and the merge of data from different sources, it has become necessary for models to support this technology. In this paper, we propose a new multidimensional model that can manage imprecision both in dimensions and facts. Consequently, the multidimensional structure is able to model data imprecision resulting from the integration of data from different sources or even information from experts, which it does by means of fuzzy logic.
\end{abstract}

\section{Introduction}

Ever since the appearance of the OLAP technology ([5]), there have been various proposals to support its special needs, and in particular, two different approaches have been documented. The first of these extends the relational model to support the structures and operations which are typical of OLAP, and the first proposal of such a type can be found in [9]. Since then, there have been other proposals (e.g. [10]), and most of the present relational systems include extensions to represent datacubes and operate on them. The second approach is to develop new models using a multidimensional view of the data. Many authors have proposed models in this way $([1,3,4,12])$.

In the early $70 \mathrm{~s}$, the need for flexible models and query languages to manage the ill-defined nature of information in DSS was identified ([8]). Nowadays, the application of the OLAP technology to other knowledge fields (e.g. medical data) and the use of semi-structured sources (e.g. XML) and non-structured sources (e.g. plain text) has made these requirements on the models even more important. The systems now need to manage imprecision in the data, and more flexible structures are needed to represent the analysis domain. New models have appeared to manage incomplete datacubes ([7]), imprecision in the facts ([11]), and the definition of facts using 
different levels in the dimensions ([13]). In addition, these models continue to use rigid hierarchies and this makes it extremely difficult for certain domains to be modelled. Consequently, this could result in the loss of information when we need to merge data from different sources with incompatibilities in their schemata.

In this paper, we propose a new multidimensional model which is able to handle imprecision in hierarchies and facts by using fuzzy logic. The use of fuzzy hierarchies enables the structures of the dimensions to be defined to the final user more intuitively, thereby allowing a more intuitive use of the system. Furthermore, this allows information to be merged from different sources with incompatibilities in their structures, or even information given by experts to be used in order to improve the multidimensional schema. In the next section, we shall introduce classical multidimensional models as an introduction to presenting our approach. Then, in the third section we shall include an example of the structure proposed to show how to apply the operations on the multidimensional structure. The final section presents the main conclusions and future work

\section{Multidimensional Model}

In this section, we shall present our proposed multidimensional model. Firstly, we shall introduce what we have called the classical models (these being the first documented models). Secondly, we shall define the multidimensional structure for managing imprecision. We shall then include the basic operations on the multidimensional models (roll-up, drill-down, dice, slice and pivot), and show how these are applied on the fuzzy structure.

\subsection{Classical Multidimensional Models}

In classical multidimensional models, we can distinguish two different types of data: on one hand, we have the facts being analysed, and on the other, the dimensions are the context for the facts. Hierarchies may be defined in the dimensions. The different levels of the dimensions allow us to access the facts at different levels of granularity. In order to do so, classical aggregation operators are needed (maximum, minimum, average, etc).

The defined hierarchies use many-to-one relations, so one element in a level can only be grouped by a single value of each upper level in the hierarchy. This makes the final structure of a datacube rigid and well defined in the sense that given two values of the same level in a dimension, the set of facts relating to these values have empty intersection.

The normal operations (roll-up, drill-down, dice, slice and pivot) are defined on this structure. 


\subsection{Multidimensional Structure}

Definition 1. A dimension is a tuple $d=\left(l, \leq_{d}, l_{\perp}, l_{\top}\right)$ where $l=\left\{l_{i}, i=1, \ldots, n\right\}$ such that each $l_{i}$ is a set of values and $l_{i} \cap l_{j}=\varnothing$ if $i \neq j$, and $\leq_{d}$ is a partial order relation between the elements of $l$. $l_{\perp}$ and $l_{\mathrm{\top}}$ are two elements in $l$ such that $\forall l_{i} \in l \quad l_{\perp} \leq_{d} l_{i}$ and $l_{i} \leq_{d} l_{\mathrm{T}}$.

Each element $l_{i}$ is called a level. In order to identify level $l$ of dimension $d$, we shall use $d . l$. The two special levels $l_{\perp}$ and $l_{\mathrm{T}}$ shall be called the base level and top level, respectively. The partial order relation in a dimension gives the hierarchical relation between the levels.

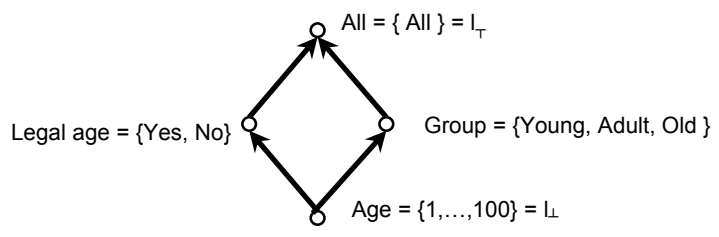

Fig. 1. Example of an age hierarchy

In Figure 1, you can see a definition of an age hierarchy. The definition of the dimension as we have presented it would be Age $=\left(\{\right.$ Age, Group, legal age, All $\}, \leq_{\text {Age }}$, Age, All), and the relation Age $\leq_{\text {Age }}$ Age, Group $\leq_{\text {Age }}$ Group, Legal age $\leq_{\text {Age }}$ Legal age, All $\leq_{\text {Age }}$ All, Age $\leq_{\text {Age }}$ Group, Age $\leq_{\text {Age }}$ Legal age, Age $\leq_{\text {Age }}$ All, Group $\leq_{\text {Age }}$ All and Legal age $\leq_{\text {Age }}$ All.

Definition 2. For each dimension $d$, the domain is $\operatorname{dom}(d)=\bigcup l_{i}$.

In the above example, the domain of the dimension Age is $\operatorname{dom}(\operatorname{Age})=\{1, \ldots, 100$, Young, Adult, Old, Yes, No, All\}.

Definition 3. For each $l_{i}$, the set

$$
H_{l_{i}}=\left\{l_{j} / l_{j} \neq l_{i} \wedge l_{j} \leq_{d} l_{i} \wedge \neg \exists l_{k} \quad l_{j} \leq_{d} l_{k} \leq_{d} l_{i}\right\},
$$

and we call this the set of children of level $l_{i}$.

Using the same example of the dimension on the ages, the set of children of the level All is $H_{A l l}=\{$ Group, Legal age $\}$. In all the dimensions we define, for the base level, this set will be always the empty set, as you can see from the definition.

Definition 4. For each $l_{i}$, the set

$$
P_{l_{i}}=\left\{l_{j} / l_{i} \neq l_{j} \wedge l_{i} \leq_{d} l_{j} \wedge \neg \exists l_{k} \quad l_{i} \leq_{d} l_{k} \leq_{d} l_{j}\right\},
$$

and we call this the set of parents of level $l_{i}$.

On the hierarchy we have defined, the set of parents of level Age is $P_{\text {Age }}=\{$ Legal age, Group $\}$. In the case of the top level of a dimension, this set will always be the empty set. 
Definition 5. For each pair of levels $l_{i}$ and $l_{j}$ such that $l_{j} \in H_{l_{i}}$, we have the relation $\mu_{i j}: l_{i} \times l_{j} \rightarrow[0,1]$, and we call this the kinship relation.

The degree of inclusion of the elements of a level in the elements of their parent levels can be defined using this relation. If we only use the values 0 and 1 and one element is only included with degree 1 for a single element of its parent levels, this relation represents a crisp hierarchy. Following the example, the relation between the levels Legal age and Age is of this type. The parent relation in this situation is

$$
\mu_{\text {Legalatge, Age }}(\text { Yes }, x)=\left\{\begin{array}{c}
1 \text { if } \mathrm{x} \in[18,100] \\
0 \text { in other case }
\end{array} \mu_{\text {Legalage,Age }}(N o, x)=\left\{\begin{array}{l}
1 \text { if } \mathrm{x} \in[1,17] \\
0 \text { in other case }
\end{array} .\right.\right.
$$

If we relax these conditions and allow values to be used in the interval $[0,1]$ without any other limitation, we have a fuzzy hierarchical relation. This allows several hierarchical relations to be represented more intuitively. An example can be seen in Figure 2 where we present the group of ages according to linguistic labels. Furthermore, this fuzzy relation allows hierarchies to be defined in which there is imprecision in the relationship between elements in different levels. In this situation, the value in the interval shows the degree of confidence in the relation.

Definition 6. For each pair of levels $l_{i}$ y $l_{j}$ of the dimension $d$ such that $l_{j} \leq_{d} l_{i} \wedge l_{j} \neq l_{i}$, the relation $\eta_{i j}: l_{i} \times l_{j} \rightarrow[0,1]$ is defined as

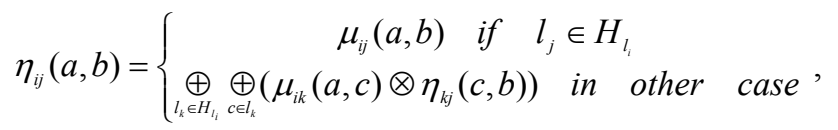

where $\oplus \mathrm{y} \otimes$ are a t-conorm and a t-norm, respectively, or operators from the families MOM or MAM defined by Yager ([15]), which include the t-conorms and t-norms, respectively. This relation is called the extended kinship relation.

This relation gives us information about the degree of relation between two values in different levels in the same dimension. In order to obtain this value, it considers all the possible paths between the elements in the hierarchy. Each one is calculated by aggregating the kinship relation between elements in two consecutive levels using a tnorm. The final value is then the aggregation of the result of each path using a tconorm. By way of example, we will show how to calculate the value of $\eta_{A l l}$, Age $(A l l, 25)$. In this situation, we have two different paths. Let us look at each:

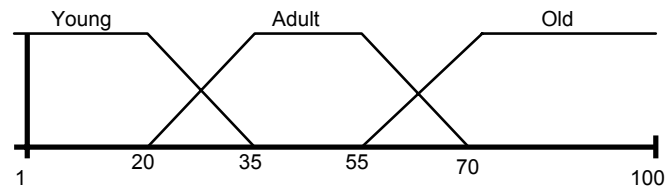

Fig. 2. Kinship relation between levels Group and Age 


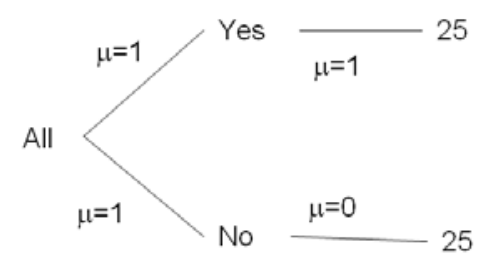

a)

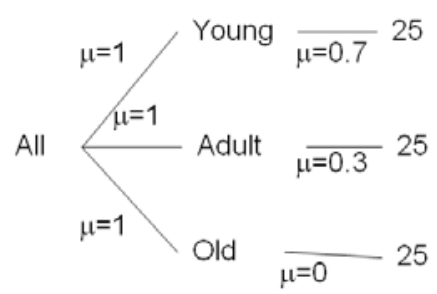

b)

Fig. 3. Example of the calculation of the extended kinship relation. a) path All - Legal ageAge b) path All - Group - Age

- $\quad$ All - Legal age - Age. In Figure 3.a, you can see the two ways to get to 25 from All passing the level legal age. The result of this path is $(1 \otimes 1) \oplus(1 \otimes 0)$.

- All-Group - Age. This situation is very similar to the previous one. In Figure 3.b, you can see the three different paths going through the level Group. The result of this path is $(1 \otimes 0.7) \oplus(1 \otimes 0.3) \oplus(1 \otimes$ $0)$.

We must now aggregate these two values using a t-conorm in order to obtain the result. If we use the maximum as the t-conorm and the minimum as the t-norm, the result is $((1 \otimes 1) \oplus(1 \otimes 0)) \oplus((1 \otimes 0.7) \oplus(1 \otimes 0.3) \oplus(1 \otimes 0))=(1 \oplus 0) \oplus(0.7 \oplus 0.3 \oplus 0)$ $=1 \oplus 0.7=1$, so the value of $\eta_{\text {All,Age }}(A l l, 25)$ is 1 , which means that the age 25 is grouped by $A l l$ in the level All with grade 1.

Definition 7. We say that any pair $(h, \alpha)$ is a fact when $h$ is an m-tuple on the attributes domain we want to analyze, and $\alpha \in[0,1]$.

The management of uncertainty in the facts is carried out using a degree of certainty with each one. This degree of certainty allows us to use values in analysis that might be interesting to the decisor but which imply imprecision. The value $\alpha$ of each pair controls the influence of the fact in the analysis.

Definition 8. An object of type history is the recursive structure

$$
H=\left\{\begin{array}{c}
\Omega \\
\left(\mathrm{A}, 1_{\mathrm{b}}, F, G, H^{\prime}\right)
\end{array},\right.
$$

where $\Omega$ is the recursivity clause, $F$ is the fact set, $l_{b}$ is a set of levels $\left(l_{l b}, \ldots, l_{n b}\right), A$ is an application from $l_{b}$ to $F, G$ is an aggregation operator, and $H^{\prime}$ is a structure of type history.

The role of this structure will be clear after the operations have been defined in the next section.

Definition 9. A datacube is a tuple $C=\left(D, l_{b}, F, A, H\right)$ such that $D=\left(d_{1}, \ldots, d_{n}\right)$ is a set of dimensions, $l_{b}=\left(l_{l b}, \ldots, l_{n b}\right)$ is a set of levels such that $l_{i b}$ belongs to $d_{i}, F=R U \varnothing$ where $R$ is the set of facts and $\varnothing$ is a special symbol, $H$ is an object of type history, and $A$ is 
an application defined as $A: l_{1 b} \times \ldots \times l_{n b} \rightarrow F$, giving the relation between the dimensions and the facts defined.

If for $\vec{a}=\left(a_{1}, \ldots, a_{n}\right), A(\vec{a})=\varnothing$, this means that no fact is defined for this combination of values.

Definition 10. We say that a datacube is basic if $l_{b}=\left(l_{1 \perp}, \ldots, l_{n \perp}\right)$ and $H=\Omega$.

Having defined the structure, we shall now show how to translate a multidimensional schema into our model. An example of a multidimensional model is shown in Figure 4. In this schema, we want to analyze the sales in a company. The broken lines represent the fuzzy relation between the levels, i.e. the relations take values in the entire interval $[0,1]$. It is possible to see how three dimensions are considered: Time, Product and Customer. This schema translated into our model corresponds to $C_{\text {sales }}=(\{$ customer, product, time $\},\{($ price, amount $)\} \cup \emptyset, A, \Omega)$. In order to complete the definition, we need the dimension structures: Customer $=(\{$ Age,

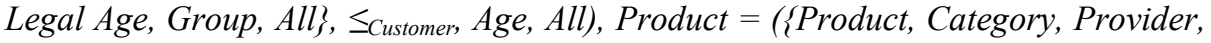
Quality, All $\}, \leq_{\text {Product }}$ Product, All $)$, Time $=\left(\{\right.$ Date, Month, Holiday, All $\}, \leq_{\text {Time }}$, Date, All) and the application $A$ that gives the relation between the dimensions and the facts: A: Age $x$ Product $x$ Date $\rightarrow\{($ price, amount $)\} \bigcup \emptyset$.

\subsection{Operations}

Once we have defined the multidimensional structure, we need the basic operations to work with it. In this section, we shall define the operations to change the level in the hierarchies (roll-up and drill-down) as well as the selection (dice), projection (slice) and pivot. First, two preliminary concepts are needed.

Definition 11. An aggregation operator is a function $G(B)$ where $B=\{(h, \alpha) /(h, \alpha) \in F\}$ and the result is a tuple $\left(h^{\prime}, \alpha^{\prime}\right)$.

The parameter of an aggregation operator can be seen as a fuzzy bag ([6]) since it concerns a collection of elements (the facts) which can be repeated, with each having a value in the $[0,1]$ interval (the $\alpha$ defined in the tuples).

Definition 12. For each value $a$ in a level $l_{i}$, we have the set

$$
F_{a}=\left\{\begin{array}{c}
\bigcup_{l_{j} \in H_{l_{i}}} F_{b} / b \in l_{j} \wedge \mu_{i j}(a, b)>0 \text { if } l_{i} \neq l_{b} \\
\left\{h / h \in H \wedge \exists a_{1}, \ldots, a_{n} A\left(a_{1}, . ., a, \ldots, a_{n}\right)=h\right\} \quad \text { if } l_{i}=l_{b}
\end{array} .\right.
$$

This set includes all the facts that are in any way related to value $a$, and this is all we need to introduce the operations and to apply them on the fuzzy multidimensional structure proposed. 


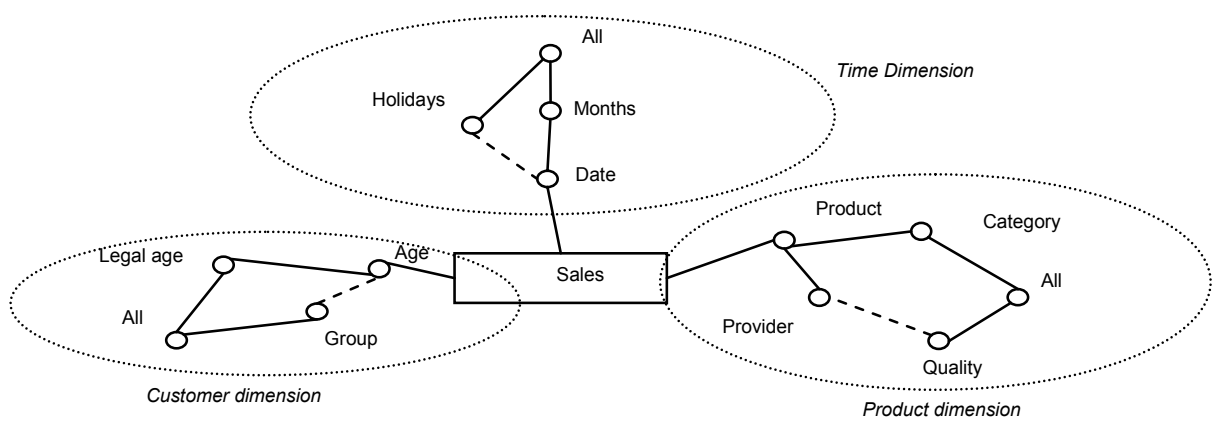

Fig. 4. Example of multidimensional schema

Definition 13. The result of applying roll-up on dimension $d_{i}$, level $l_{r}\left(l_{r} \neq l_{\perp}\right)$, using the aggregation operator $\mathrm{G}$ on a datacube $C=\left(D, l_{b}, F, A, H\right)$ is another datacube $C^{\prime}=\left(D, l_{b}^{\prime}, F^{\prime}, A^{\prime}, H^{\prime}\right) \quad$ where $\quad l^{\prime}{ }_{b}=\left(l_{l b}, \ldots, l_{r}, \ldots, l_{n b}\right)$, $A^{\prime}\left(a_{1}, \ldots, a, \ldots, a_{n}\right)=G\left(\left\{\left(b, \alpha \otimes \eta_{r b}(a, c)\right) /(b, \alpha) \in F_{a} \wedge A\left(a_{1}, \ldots, c, \ldots, a_{n}\right)=(b, \alpha)\right\}\right), F^{\prime}$ is the range of $A^{\prime}$, and $H^{\prime}=\left(A, l_{b}, F, G, H\right)$.

Definition 14. The result of applying drill-down on a datacube $C=\left(D, l_{b}, F, A, H\right)$ having $H=\left(A^{\prime}, l_{b}, F^{\prime} . H^{\prime}\right)$ is another datacube $C^{\prime}=\left(D, l_{b}, F^{\prime}, A^{\prime}, H^{\prime}\right)$.

After the definition of the drill-down operation, we can see the role of the structure history inside our proposal. This recursive structure enables us to return at any time to the previous state before the roll-up was applied. Consequently, loss of information is prevented as you progress up the hierarchy.

Definition 15. The result of applying dice with the condition $\beta$ on level $l_{r}$ of dimension $d_{i}$ in a datacube $C=\left(D, l_{b}, F, A, H\right)$ is another datacube $C^{\prime}=\left(D^{\prime}, l^{\prime}{ }_{b}, F^{\prime}, A^{\prime}, \Omega\right)$ where $D^{\prime}=\left\{d_{1}, \ldots, d^{\prime}{ }_{i}, \ldots, d_{n}\right\}$ where $d_{i}{ }^{\prime}=\left(l_{i}{ }^{\prime}, \leq_{d i}, l_{b}, l_{T}\right)$ having $l^{\prime}=\left\{l_{j} l_{b} \leq d i l_{j}\right\}$ and

$$
\begin{aligned}
& d_{i}^{\prime} l_{j}^{\prime}=\left\{\begin{array}{cl}
\left\{v / v \in l_{j} \wedge \beta(v)\right\} & \text { if } l_{j}^{\prime}=l_{r} \\
\left\{v / v \in d_{i} \cdot l_{j} \wedge \exists x \in l_{r} \beta(x) \wedge \eta_{r j}(x, v)>0\right\} & \text { if } l_{j}^{\prime} \leq_{d} l_{r}, \\
\left\{v / v \in d_{i} \cdot l_{j} \wedge \exists x \in l_{r} \beta(x) \wedge \eta_{j r}(v, x)>0\right\} & \text { if } l_{r} \leq_{d} l_{j}^{\prime}
\end{array}\right. \\
& A^{\prime}\left(a_{1}, \ldots, a_{i}, \ldots, a_{n}\right)=\left(h, \alpha \otimes \mu_{\beta}\right) / a_{1} \in d_{1}^{\prime} \cdot l_{b}^{\prime} \wedge \ldots a_{n} \in d_{n}^{\prime} l_{b}^{\prime} \wedge A\left(a_{1}, \ldots, a_{n}\right)=(h, \alpha) \quad \text { where } \\
& \mu_{\beta}=\underset{c \in d_{r}^{\prime} \cdot l_{r}^{\prime}}{\oplus} \eta_{r b}\left(c, a_{i}\right), \text { and } F^{\prime} \text { is the range of } A^{\prime} .
\end{aligned}
$$

Definition 16. The result of applying slice on dimension $d_{i}$ using the aggregation operator $G$ in a datacube $C=\left(D, l_{b}, F, A, H\right)$ is another datacube $C^{\prime}=\left(D^{\prime}, l_{b}{ }^{\prime}, F^{\prime}, A^{\prime}, \Omega\right)$ where $\quad D^{\prime}=\left(d_{1}, \ldots, d_{i-1}, d_{i+1}, \ldots, d_{n}\right), \quad l_{b}{ }^{\prime}=\left(l_{i b}, \ldots, l_{i-1 b}, l_{i+l b}, \ldots, l_{n b}\right)$, $A^{\prime}\left(a_{1}, \ldots, a_{i-1}, a_{i+1}, \ldots, a_{n}\right)=G\left(\left\{(h, \alpha) / \exists x A\left(a_{1}, \ldots, a_{i-1}, x, a_{i+1}, \ldots, a_{n}\right)=(h, \alpha)\right\}\right)$, and $F^{\prime}$ is the range of $A^{\prime}$.

Definition 17. The result of applying pivot on dimensions $\mathrm{d}_{\mathrm{i}}$ and $\mathrm{d}_{\mathrm{j}}$ in a datacube $C=\left(D, l_{b}, F, A, H\right) \quad$ is another datacube $C^{\prime}=\left(D^{\prime}, l_{b}, F, A^{\prime}, \Omega\right)$ where $D^{\prime}=\left(d_{1}, \ldots, d_{i-1}\right.$ 
$\left., d_{j}, d_{i+1}, \ldots, d_{j-1}, d_{i}, d_{j+1}, \ldots, d_{n}\right), \quad l_{b}{ }^{\prime}=\left(\quad l_{1 b}, \ldots, l_{i-1 b}, l_{j b}, l_{i+l b}, \ldots, l_{j-1 b}, l_{i b}, l_{j+l b}, \ldots, l_{n b}\right), \quad$ and $A^{\prime}\left(a_{1}, \ldots, a_{i-1}, a_{i}, a_{i+1}, \ldots, a_{j-1}, a_{j}, a_{j+1}, \ldots, a_{n}\right)=A\left(a_{1}, \ldots, a_{i-1}, a_{j}, a_{i+1}, \ldots, a_{j-1}, a_{i}, a_{j+1}, \ldots, a_{n}\right)$.

Although we now have the operations to work with the structure proposed, this structure can represent objects that are not suitable for the operations defined above. We must therefore say when a datacube is valid to work with it.

Definition 18. A datacube is valid if it is basic or has been obtained by applying a finite number of operations on a basic datacube.

\subsection{User View}

We have presented a structure that manages imprecision by means of fuzzy logic. We need to use aggregation operators on fuzzy bags in order to apply some of the operations presented. Most of the methods previously documented give a fuzzy set as a result. As this situation can make the result difficult to understand and use in a decision process, we propose a two-layer model: one of the layers is the structure presented in the previous section; and the other is defined on this, and its main objective is to hide the complexity of the model and provide the user with a more understandable result. In order to do so, we propose the use of a fuzzy summary operator that gives a more intuitive result but which keeps as much information as possible. Using this type of operator, we shall define the user view.

Definition 19. Given a summary operator $M$, we define the user view of a datacube $C=\left(D, l_{b}, F, A, H\right) \quad$ using $\quad M$ as the structure $C_{M}=\left(D, l_{b}, F_{M}, A_{M}\right) \quad$ where $A_{M}\left(a_{1}, \ldots, a_{n}\right)=M\left(A\left(a_{1}, \ldots, a_{n}\right)\right)$ and $F_{M}$ is the range of $A_{M}$.

We can define as many user views of a datacube as the number of summary operators used. Therefore, each user can have their own user view with the most intuitive view of data according to their preferences by using a datacube. As an example of this type of operator, we can use the one proposed in [2]. This operator proposes the use of the fuzzy number that best fits, in the sense of fuzziness, the fuzzy set or fuzzy bag.

\section{Example}

Once we have defined the fuzzy structure and the operations on it, we shall present an example of a simple multidimensional schema in order to show the application of operations on it. This example will be modelled using the classical multidimensional or crisp model to show the differences between both approaches. We will use the schema in Figure 4.

In the fuzzy case, the dimension Customer is the fuzzy hierarchy on ages which we have used previously. The remaining elements in both the fuzzy and the crisp case are shown in Figure 5, with the exception of the partial order relations which are clear in the schema. Here we see the first differences between both approaches when we model the levels group and holiday. In the crisp case, these concepts are modelled 
using intervals on the ages and dates, respectively. In our approach, we use linguistic labels. The facts used in the example and their relation with the values in the dimension are shown in Table 1. If the user wants to know "the average amount of sales at Christmas for the different age groups and the quality of the provider", the sequence of operations to apply is:

1. dice on the dimension time, in the level holiday with the condition $\beta(x)=$ " $x$ is Christmas".

2. roll-up in the dimension time and level holiday, dimension product and level quality and dimension customer and level group, using the aggregation operator average on the amount.

Time

All $=\{$ All $\}=I_{T}$ Moths $=\{$ Dec $-02, \ldots$, Jan -03$\} \quad$ Holiday $=\{$ Chistmas $\} \quad F$ echas $=\{01-$ dec $-02, \ldots, 31-$ Jan -03$\}=I_{\perp}$ Product

$A \| l=\{A \| l\}=I_{T}$ Category $=\{$ milk, other $\}$ Provider $=\{P 1, P 2, P 3\}$ Quality $=\{$ Good,Medium, Bad $\}$ Product $=\{$ milk, bread, juice, cheese, meat $\}=I_{\perp}$

\section{Crisp Model}

\begin{tabular}{|l|l|}
\hline Group & Ages \\
\hline Young & {$[0,25]$} \\
\hline Adult & ] $25,65[$ \\
\hline Old & {$[65,100]$} \\
\hline
\end{tabular}

Fuzzy Model

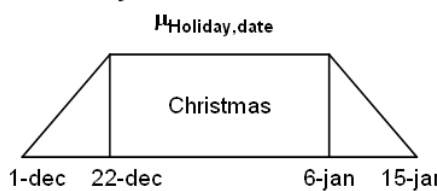

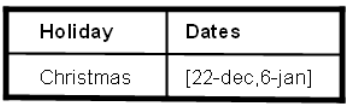

\begin{tabular}{|l|l|}
\hline Quality & Providers \\
\hline Good & P1 \\
\hline Medium & P2 \\
\hline Bad & P3 \\
\hline
\end{tabular}

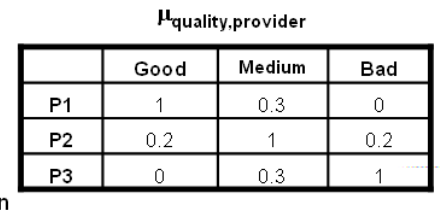

$\mu_{\text {provider,product }}$

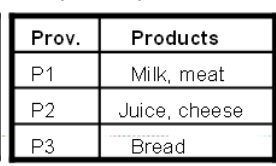

Fig. 5. Dimension structures for the multidimensional schema

In order to apply the roll-up operation, we need the average aggregation operator. Although we can use the classical operator in the crisp case, in the fuzzy model we need an operator that works with fuzzy bags. In the example, we have used the operators proposed by Rundensteiner ([14]) for a fuzzy relational model. The adaptation of these operators to our approach is simple: if $R$ is an aggregation operator defined by Rundensteiner, the operator $G_{R}$ for our approach is defined as $G_{R}(h)=(R(h), 1)$.

We need another operator to show the results in the fuzzy case. We have used the linguistic summary ([2]) as the summary operator. The results in both approaches are shown in the Tables 2-4. When analyzing the results, we need to bear in mind the differences between both approaches. Therefore, when the user gets the result in the crisp case, for example for the group young, the results correspond to the query "the average amount of sales in the interval [22-dic,6-jan] by the customer with ages in the interval [0,25] and the quality of the provider". In the fuzzy case, the user gets a result which is closer to his/her concept of Christmas and youth. 
If we want to refine the results in order to obtain "the maximum average amounts sold by age groups", we need to apply slice on the dimensions Products and Time, using the maximum aggregation operator. The result is shown in Table 5.

The results obtained in each case are different. This occurs because the values involved in each calculation and their importance are different in both approaches. In the crisp case, all the values inside the intervals have the same weight in the aggregation process. In the fuzzy model, on the other hand, the values at the edges of the concepts do not have the same importance as the values in the kernel in the final result. We can also see the role of the user view in the fuzzy model. The multidimensional structure proposed is based on fuzzy logic and the results shown to the user are fuzzy sets which are difficult to understand. The user view helps to interpret the results, showing the information obtained in a more expressive and understandable way to the user (using a fuzzy number and the associated linguistic expression in each case).

Table 1. Data in the datacube example

\begin{tabular}{|c|c|c|c|c|c|c|c|c|c|c|c|c|c|}
\hline 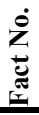 & 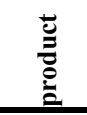 & 气̃ & $\underset{<}{\infty}$ & 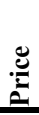 & $\stackrel{\Xi}{\Xi}$ & $\alpha$ & 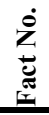 & 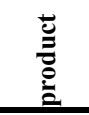 & $\begin{array}{l}\stackrel{\Xi}{*} \\
\text { D }\end{array}$ & $\underset{0}{8}$ & $\stackrel{\mathscr{e}}{=}$ & $\stackrel{\Xi}{\Xi}$ & $\alpha$ \\
\hline 1 & milk & 23-dec & 19 & 10 & 1 & 1 & 13 & bread & 6-jan & 17 & 3 & 2 & 1 \\
\hline 2 & meat & 7-jan & 40 & 18 & 3 & 1 & 14 & meat & 22-dec & 65 & 6 & 3 & 1 \\
\hline 3 & bread & 10-jan & 45 & 1 & 5 & 1 & 15 & cheese & 2-jan & 52 & 10 & 2 & 1 \\
\hline 4 & juice & $28-\mathrm{dec}$ & 75 & 2 & 2 & 1 & 16 & bread & $27-\mathrm{dec}$ & 66 & 5 & 2 & 1 \\
\hline 5 & cheese & 3-jan & 20 & 5 & 1 & 1 & 17 & cheese & 04-jan & 70 & 5 & 3 & 1 \\
\hline 6 & milk & 10-jan & 20 & 1 & 5 & 1 & 18 & bread & 24-dec & 60 & 3 & 6 & 1 \\
\hline 7 & bread & $25-\mathrm{dec}$ & 22 & 3 & 1 & 1 & 19 & bread & 10-jan & 65 & 4 & 4 & 1 \\
\hline 8 & bread & 1-jan & 55 & 5 & 2 & 1 & 20 & milk & 03-jan & 64 & 5 & 2 & 1 \\
\hline 9 & juice & $28-\mathrm{dec}$ & 23 & 4 & 3 & 1 & 21 & cheese & 10-jan & 15 & 5 & 5 & 1 \\
\hline 10 & bread & 6-jan & 75 & 6 & 4 & 1 & 22 & cheese & 28-dec & 40 & 3 & 5 & 1 \\
\hline 11 & milk & 23-dec & 78 & 3 & 3 & 1 & 23 & bread & 02-jan & 65 & 4 & 5 & 1 \\
\hline 12 & meat & 29-dec & 40 & 18 & 2 & 1 & 24 & milk & 26-dec & 23 & 5 & 5 & 1 \\
\hline
\end{tabular}

\section{Conclusions}

In this paper, we have presented a new multidimensional model. The main contribution of this new model is that it is able to operate on data with imprecise facts and hierarchies. Classical models impose a rigid structure that makes it difficult for information from different sources to be merged if there are incompatibilities in the schemata. Our model can handle these problems by means of fuzzy logic which allows our proposal to carry out the integration, relaxing the schemata in order to obtain a new one that covers the others and attempting to preserve as much information as possible. In addition, our model can manage information given by experts which is often imprecise. This data can be used to improve the multidimensional schema so that it may be used by the final user in the decision process. Another advantage is that it can model situations to users more naturally so that they can access the information more intuitively. 
Table 2. Result of applying dice on the dimension Time, on the level Holiday with the condition $\beta(x)=$ " $x$ is Christmas " over C. In the fuzzy case, the value shown is the new $\alpha$ of the fact. In the crisp case, $\mathrm{X}$ means that this fact satisfies the condition.

\begin{tabular}{|c|c|c|c|c|c|c|c|c|c|c|c|c|}
\hline Fact & 1 & 2 & 3 & 4 & 5 & 6 & 7 & 8 & 9 & 10 & 11 & 12 \\
\hline Fuzzy & 1 & 0.9 & 0.6 & 1 & 1 & 0.6 & 1 & 1 & 1 & 1 & 1 & 1 \\
\hline Crisp & X & - & - & $X$ & $X$ & - & $X$ & $X$ & $X$ & $X$ & $X$ & $X$ \\
\hline Fact & 13 & 14 & 15 & 16 & 17 & 18 & 19 & 20 & 21 & 22 & 23 & 24 \\
\hline & 1 & 1 & 1 & 1 & 1 & 1 & 0.6 & & 0.6 & 1 & 1 & 1 \\
\hline Crisp & X & $X$ & $X$ & $X$ & $X$ & - & $X$ & $\mathrm{X}$ & - & $X$ & $X$ & X \\
\hline
\end{tabular}

Table 3. Result of applying roll-up in the dimension Time on the level Holidays, dimension Product and level Quality and dimension Customer and level Group in the datacube C' in the fuzzy case. Time dimension is not shown due to the fact that there is only one value

\begin{tabular}{|c|c|c|c|c|c|c|}
\hline \multirow{3}{*}{ Customer } & \multicolumn{6}{|c|}{ Product } \\
\hline & \multicolumn{2}{|c|}{ Good } & \multicolumn{2}{|c|}{ Medium } & \multicolumn{2}{|c|}{ Bad } \\
\hline & C"' & $C{ }^{\prime}{ }_{M}$ & C"' & $C{ }^{\prime}{ }_{M}$ & C"' & $C{ }^{\prime}{ }_{M}$ \\
\hline Young & $\begin{array}{c}\{1 / 10.6 / 3 \\
0.4 / 3.67 \\
0.2 / 3.33\}, 1\end{array}$ & $\begin{array}{l}(1,1,0,1.5) \\
\text { "greater } \\
\text { than 1" }\end{array}$ & $\begin{array}{l}\{1 / 1,0.6 / 3, \\
0.3 / 2.88\}, 1\end{array}$ & $\begin{array}{c}(1,1,0,1.45) \\
\text { "greater than } \\
1 "\end{array}$ & $\begin{array}{c}\{1 / 2, \\
0.6 / 1.5 \\
0.2 / 2.4\}, 1\end{array}$ & $\begin{array}{c}(2,2,0.5,0.39) \\
\text { "around 2" }\end{array}$ \\
\hline Adult & $\begin{array}{c}11 / 2 \\
0.9 / 2.5 \\
0.6 / 3.4 \\
0.5 / 3.33 \\
0.2 / 3.3\}, 1\end{array}$ & $\begin{array}{l}(2,2,0,1.19) \\
\text { "greater } \\
\text { than 2" }\end{array}$ & $\begin{array}{c}\{1 / 3.5 \\
0.6 / 3.33 \\
0.3 / 3.44\}, 1\end{array}$ & $\begin{array}{c}(3.5,3.5,0.17,0) \\
\text { "a bit less than } \\
3.5 "\end{array}$ & $\begin{array}{c}\{1 / 2,0.8 / 4 \\
0.5 / 3.8 \\
0.4 / 3.33 \\
0.2 / 3.3\}, 1\end{array}$ & $\begin{array}{c}(2,2,0,1.6) \\
\text { "grater than } \\
2 "\end{array}$ \\
\hline Old & $\begin{array}{c}\{1 / 3 \\
0.5 / 2.67 \\
0.2 / 2.6\}, 1\end{array}$ & $\begin{array}{l}(3,3,0.4,0) \\
\text { "a bit less } \\
\text { than 3" }\end{array}$ & $\begin{array}{c}\{1 / 2, \\
0.8 / 2.5 \\
0.3 / 3.22\}, 1\end{array}$ & $\begin{array}{c}(2,2,0,1.22) \\
\text { "greater than } \\
2 "\end{array}$ & $\begin{array}{c}\{1 / 4,0.6 / 3 \\
0.5 / 3.75 \\
0.3 / 4.2 \\
0.2 / 3.71\}, 1\end{array}$ & $\begin{array}{c}(4,4,0.29, \\
0.19) \\
\text { "around } 4 "\end{array}$ \\
\hline
\end{tabular}

Table 4. Result of applying roll-up in the dimension Time on the level Holiday, dimension Product and level Quality and dimension Customer and level Group in the datacube C' in the crisp case

\begin{tabular}{|c|c|c|c|}
\cline { 2 - 4 } \multicolumn{1}{c|}{} & \multicolumn{3}{c|}{ Product } \\
\hline Customer (Age group) & Good & Medium & Bad \\
\hline Young & 3 & 2 & 1.5 \\
\hline Adult & 2 & 3.5 & 4 \\
\hline Old & 3 & 2.5 & 3.7 \\
\hline
\end{tabular}

Table 5. Result of applying slice on the dimensions Product and Time in the datacube C",

\begin{tabular}{|c|c|c|c|}
\hline & \multicolumn{2}{|l|}{ Fuzzy } & Crisp \\
\hline Customer & C"” & $C,{ }_{M}$ & Fact \\
\hline Young & $\begin{array}{c}\{1 / 2,0.6 / 1.5,0.2 / 2.4,0.6 / 3,0.3 / 2.88 \\
0.4 / 3.67,0.2 / 3.33\}, 1\end{array}$ & $\begin{array}{l}(2,2,0.5,1.3) \\
\text { "around 2" }\end{array}$ & 3 \\
\hline Adult & $\begin{array}{c}\{1 / 3.5,0.8 / 4,0.6 / 3.8,0.6 / 3.33,0.3 / 3.44 \\
0.5 / 3.67\}, 1\end{array}$ & $\begin{array}{l}(3.5,3.5,0.17,0.5) \\
\text { "around 3.5" }\end{array}$ & 4 \\
\hline Old & $\begin{array}{c}\{1 / 4,0.6 / 3,0.5 / 3.75,0.3 / 4.2,0.2 / 3.71 \\
0.3 / 3.22\}, 1\end{array}$ & $\begin{array}{l}(4,4,0.99,0.2) \\
\text { "around 4" }\end{array}$ & 3,7 \\
\hline
\end{tabular}

In order to complete the model, we need to study the properties of the operations on the structure. Another line is to develop a graphical means of representing the results of the operations so that the information obtained may be read more 
intuitively. To finish the decision process, we need to study the integration process so as to obtain a formal way to merge data from different sources, including experts' knowledge.

\section{References}

1. Agrawal, R. Gupta, A., Sarawagi, S.: Modeling Multidimensional Databases. IBM Research Report, IBM Almaden Research Center, September 1995

2. Blanco, I., Sánchez, D., Serrano, J.M., Vila, M.A.: A New Proposal of Aggregation Functions: the Linguistic Summary. Proceedings of IFSA'2003 Istanbul (Turkey) 2003

3. Cabibbo, L., Torlone, R.: A Logical Approach to Multidimensional Databases. Advances in Databases Technology (EDTB'98) No. 1337 in LNCS pp. 183-197 Springer 1998

4. Cabibbo, L., Torlone, R..: Querying Multidimensional Databases. Proceedings of the $6^{\text {th }}$ Int. Workshop on databases programming languages (DBPL6) Estes Pork (U.S.A.) 1997

5. Codd, E.F.: Providing OLAP (On-line Analytical Processing) to User-Analysts: An IT Mandate. Technical report, E.F. Codd and Associates, 1993

6. Delgado, M., Martín-Bautista, M.J., Sánchez, D., Vila, M.A.: On A Characterization of Fuzzy Bags. Proceedings of IFSA'2003 Istanbul (Turkey) 2003

7. Dyreson, C.: Information Retrieval from an Incomplete Data Cube. Proceedings of the $22^{\text {nd }}$ Int. Conf. on VLDB pp. 532-543. Morgan Kaufman Publishers, 1996

8. Gorry, G.A., Scott Morton, M.S.: A Framework for Management Information Systems. Sloan Management Review 13 (1) (1971) 50-70

9. Gray, J., Chaudhuri, S., Bosworth, A., Layman, A., Reichart, D., Venkatrao, M.: Data Cube: A Relational Aggregation Operator Generalizing Group-By, Cross-Tab, and Sub-Totals. Data Mining and Knowledge Discovery 1 (1997) 29-53

10. Kimball, R.: The Data Warehouse Toolkit. Wiley, New York, 1996

11. Laurent, A., Bouchon-Meunier, B., Doucet, A.: Flexible Unary Multidimensional Queries and their Combinations. Proceedings of IPMU 2002, Annecy (France) 2002

12. Li, C., Wang, X.S.: A Data Model for Supporting On-Line Analytical Processing. Proceedings of the $5^{\text {th }}$ Int. Conf. on Information and Knowledge Management (CIKM) 1996

13. Pedersen, T.B., Jensen, C.S., Dyreson, C.E.: A Foundation for Capturing and Querying Complex Multidimensional Data. Information Systems 26 (2001) 383-423

14. Rundensteiner, E.A., Bic, L.: Aggregates in Possibilistic Databases. Proceedings of the $15^{\text {th }}$ Conf. on Very Large Databases (VLDB'98), Amsterdam (Holland), 287-295, 1989

15. Yager, R.R.: Aggregation Operators and Fuzzy Systems Modelling. Fuzzy Sets and Systems 67 (1994) 129-145 